\title{
Broadband III-V on silicon hybrid superluminescent LEDs by quantum well intermixing and multiple die bonding
}

\author{
A. De Groote ${ }^{1,2, *}$, J.D. Peters ${ }^{1}$, M.L. Davenport ${ }^{1}$, M.J.R. Heck ${ }^{1}$, R. Baets ${ }^{2}$, G. Roelkens ${ }^{2}$ and J.E. Bowers ${ }^{1}$ \\ ${ }^{1}$ Optoelectronics Research Group, ECE Department, University of California, Santa Barbara, United States \\ ${ }^{2}$ Photonics Research Group, Department of Information Technology, Ghent University - imec, Ghent, Belgium \\ *Email: andreasdegroote@intec.ugent.be
}

\begin{abstract}
Combining quantum well intermixing and multiple die bonding a broadband superluminescent III-V on silicon LED was realized. Balancing four LEDs with different band gaps resulted in $292 \mathrm{~nm} 3 \mathrm{~dB}$ bandwidth and an on-chip power of $-8 \mathrm{dBm}$.
\end{abstract}

\section{INTRODUCTION}

While originally conceived for data and telecom applications, silicon photonics is rapidly emerging as a potential integration platform for a broader range of applications. Using standard CMOS fabrication techniques the silicon fabrication can achieve very high yield. However, it is almost impossible to make a light source on silicon due to its indirect band gap. Therefore III-V compounds such as InP are integrated on silicon-on-insulator (SOI) waveguide circuits. In these so-called heterogeneously integrated devices the gain is provided by the active region grown on a III-V substrate. [1] [2] [3]

Superluminescent diodes (SLDs) are suitable for numerous applications ranging from optical component testing to sensing applications (e.g. gyroscopes) as well as medical imaging (e.g. optical coherence tomography). For these applications, large bandwidth is of critical importance. There have been different approaches of extending the bandwidth of the III-V gain medium, among which dual quantum well design [4], multi-state quantum wells [5] and quantum dots [6]. These structures are however difficult to design and optimize and operate well only using specific drive currents. Alternatively, broadband supercontinuum sources can be considered, but these typically require ultra-short pulse sources that cannot yet be integrated on the silicon-on-insulator waveguide circuit [7].

To solve these limits, we designed and demonstrated a superluminescent single mode light emitting diode with different active sections having a different band gap, integrated on a silicon waveguide circuit. Thanks to the implantation enhanced disordering quantum well intermixing technique (IED-QWI), we can blueshift certain areas of the to-be-bonded InP die. On top of that, we can bond several dies on one SOI waveguide circuit. To our knowledge, it is the first time ever that these two techniques have been combined, although they are very complementary. With this achievement, we merged four different band gaps in a serial manner, as indicated in figure 1 .

\section{DEVICE DESIGN}

Since two epitaxial designs are bonded side by side, we have a large design freedom for the epitaxial layer stack. We used the same layer stack as described in [8], be it one with QWs providing gain around $1380 \mathrm{~nm}$ and one around $1540 \mathrm{~nm}$. The downside of this multiple die bonding is the reduced flexibility in the positioning of the different materials. If one would only use this technique, four different dies have to be bonded next to each other. Consequently the device area grows very large. Quantum well intermixing is a technique of changing the composition of the quantum wells by atom disordering.
As this is lithographically defined, it does give us this extra flexibility and makes it possible to have two band gaps in the same mesa. The maximum shift is limited to $\pm 100 \mathrm{~nm}$ around telecom wavelengths though.

Because of this, our structures were designed such that each device has two mesas, each consisting of two band gaps, as indicated in figure 1. The narrowest band gap is utmost left, the largest is

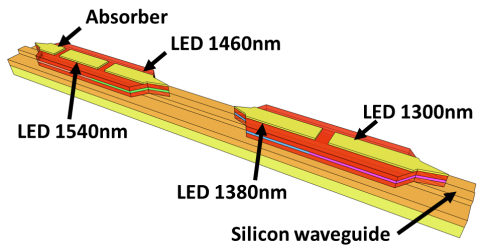

(a) $3 \mathrm{D}$ overview

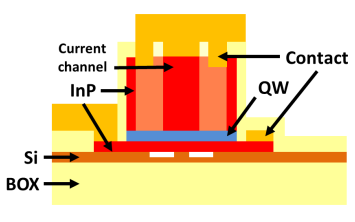

(b) Cross section of the III$\mathrm{V}$ on silicon waveguide.
Fig. 1: Illustration of the broadband LED

utmost right. This way the light can travel towards the right without suffering from strong band-to-band absorption. Any photons traveling towards the left are absorbed by the narrower band gaps, thereby optically pumping the narrow band gap material. In this manner, we have created a one-directional device which only emits on the right side. In the design of an LED, special precautions have to be taken to prevent lasing, which would narrow the spectrum and increase the coherence length. Here, inherently it is impossible to lase for all band gaps because the band gap to the left will always heavily absorb the generated light, so no net roundtrip gain is possible. This is true for all band gaps except the smallest one. Therefore, we created an extra absorbing section on the left side.

\section{DEVICE FABRICATION}

Fabrication starts with the preparation of two InP samples. First alignment markers are etched, in order to be able to do an aligned bonding. The to-be-intermixed areas are implanted with phosphor ions into the buffer layer. Subsequently, they are propagated into the quantum wells during a $700 \mathrm{C}$ thermal anneal. Because phosphor starts to evaporate at 500C at atmospheric pressure [9], a cap layer is necessary (a $\mathrm{SiN} / \mathrm{SiO} 2 / \mathrm{SiN}$ strain compensated layer).

In the SOI sample, trenches of $250 \mathrm{~nm}$ deep are dry etched in the $500 \mathrm{~nm}$ thick silicon device layer to form the waveguide, followed by the etching of vertical outgassing channels, which increase the bonding yield. Plasma-assisted bonding is carried out as described in [10]. Note that in this work, the SOI and InP dies have to be aligned during bonding using a flip chip bonder. An alignment error of $2 \mu \mathrm{m}$ was achieved. After die attachment, both dies are annealed 
at the same time, creating the strong covalent bonds. Because the post bond process contains a few dry etches which might damage the silicon waveguides in between the dies, $1 \mu \mathrm{m}$ low-temperature PECVD $\mathrm{SiO}_{2}$ was deposited. This forms a thick barrier both for the damage of dry etches as well as for preventing wet etchants to enter the waveguide trenches. After this, the InP substrates are removed.

The post bond process starts with mesa definition. A RIE etching was used to etch down into the QW layer, and then a selective wet etch was used to stop on the n-InP layer. $\mathrm{Pd} / \mathrm{Ti} / \mathrm{Pd} / \mathrm{Au}$ and $\mathrm{Pd} / \mathrm{Ge} / \mathrm{Pd} / \mathrm{Au}$ were deposited as contact metals for the $\mathrm{p}$ - and n-contacts respectively. The contact resistivity of these contacts was measured with the transmission line method (TLM) to be $3 \times 10^{-5} \Omega \mathrm{cm}^{2}$ for the pcontact, and $4 \times 10^{-7} \Omega \mathrm{cm}^{2}$ for the n-contact. The mesa was $24 \mu \mathrm{m}$ wide, so proton implantation was necessary to form a $4 \mu \mathrm{m}$ wide current channel. Finally, the different sections are isolated from each other, by etching the p-contact layer (InGaAs) and part of the p-InP. This rendered an electrical isolation of more than $50 \mathrm{k} \Omega$. Note that both bonded dies are processed at the same time. [11]

The tapers used to efficiently couple light from the III-V on silicon hybrid structure to the passive silicon waveguide are $20 \mu \mathrm{m}$ long and are 2-staged, with a narrow p-InP layer and a gradual taper in the quantum wells. The length of each section is $1 \mathrm{~mm}$.

\section{RESULTS}

Figure 2 shows the $\mathrm{CW}$ performance of the fabricated device at 20C. Each section can be controlled separately, with the different Pcontacts being isolated by more than $50 \mathrm{k} \Omega$. From the test structure, we know the output power of each section separately is very similar. Hence, from figure $2 \mathrm{a}$ we conclude that there is considerable loss in the system. The longest wavelengths have to travel furthest through the structures, and their power is lowest.

The different currents are chosen such that we have a broad, flattop spectrum as shown in figure $2 \mathrm{~b}$ The pump current was $70 \mathrm{~mA}$, $50 \mathrm{~mA}, 300 \mathrm{~mA}$ and $140 \mathrm{~mA}$ for the sections at $1300 \mathrm{~nm}, 1380 \mathrm{~nm}$, $1460 \mathrm{~nm}$ and $1540 \mathrm{~nm}$ respectively. A total on-chip power of $-8 \mathrm{dBm}$ is achieved with a $3 \mathrm{~dB}$ bandwidth of $292 \mathrm{~nm}$. The O,E,S and part of the $\mathrm{C}$ band were covered, ranging from $1258 \mathrm{~nm}$ to $1550 \mathrm{~nm}$.

It can be concluded that the loss, which was observed in figure $2 \mathrm{a}$ is mainly due to residual absorption in the quantum wells the light has to travel through. When all sections are pumped, the difference in power is nearly gone.

From the dotted line in figure $2 \mathrm{a}$ one may conclude that that section emits at $1580 \mathrm{~nm}$ rather than $1540 \mathrm{~nm}$. This is contradicted by our measurement of the backside emission of the absorbing section, which does show a maximum at $1540 \mathrm{~nm}$ (not shown here). Also here the residual absorption is to blame. Since this absorption is below band gap absorption, it is highly dependent on the wavelength. The shorter wavelengths are much more absorbed than the longer ones. Light is generated with a maximum around $1540 \mathrm{~nm}$, but the shorter wavelengths are absorbed. Since we measure the product of both emission and absorption the measured maximum is $1580 \mathrm{~nm}$ rather than $1540 \mathrm{~nm}$. A similar behaviour is seen in figure $2 \mathrm{~b}$ where the maximum at $1500 \mathrm{~nm}$ is highly dependent on the pumping conditions of both sections. Instead of absorption, there is below band gap gain. This highly asymmetric gain profile amplifies the shorter wavelengths much more than the longer, causing the maximum to shift to $1500 \mathrm{~nm}$ rather than $1540 \mathrm{~nm}$.

\section{SUMMARY}

For the first time ever, quantum well intermixing and multiple die bonding were combined. In this manner, we have realized a broadband LED on silicon. Four different band gaps were created and merged in order to cover the O,E,S and $\mathrm{C}$ band. The device was designed to be a one-directional device by arranging the band gaps from narrow to wide. When pumping the different sections to achieve a flat top

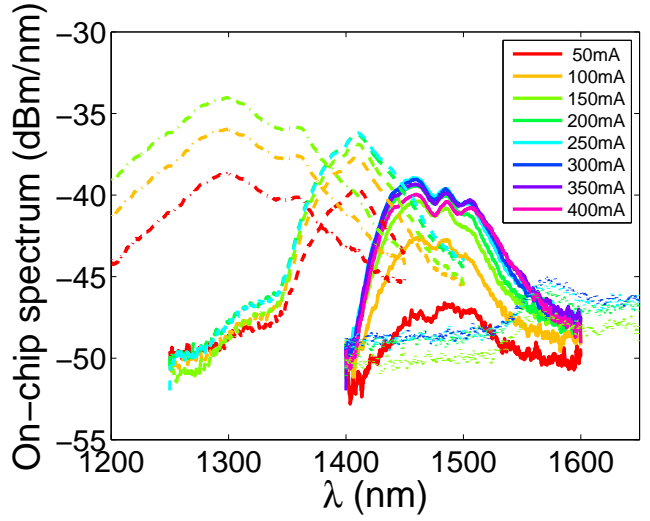

(a) Different sections pumped separately. We used a dashed, dashdotted, full and dotted line for the section at $1300 \mathrm{~nm}, 1380 \mathrm{~nm}$, $1460 \mathrm{~nm}$ and $1540 \mathrm{~nm}$ respectively.

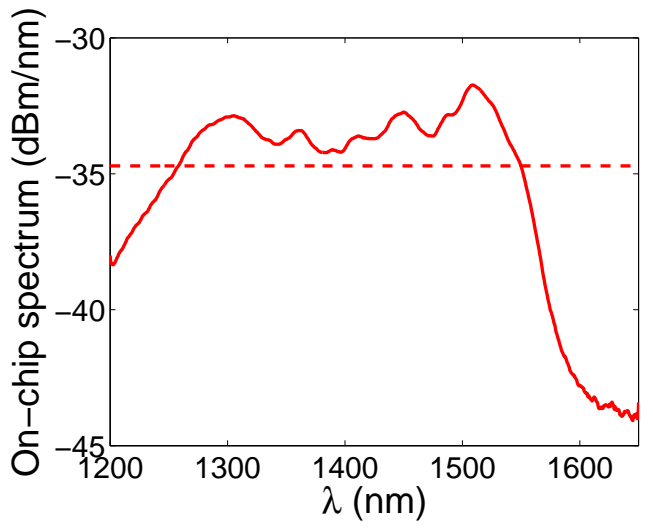

(b) Balanced pumping to optimize the $3 \mathrm{~dB}$ band width. The pumping currents were $70 \mathrm{~mA}, 50 \mathrm{~mA}, 300 \mathrm{~mA}$ and $140 \mathrm{~mA}$ for the sections at $1300 \mathrm{~nm}, 1380 \mathrm{~nm}, 1460 \mathrm{~nm}$ and $1540 \mathrm{~nm}$ respectively.

Fig. 2: On-chip spectra of the depicted device.

spectrum, we managed to achieve $292 \mathrm{~nm} 3 \mathrm{~dB}$ bandwidth, while the total power was $-8 \mathrm{dBm}$.

This research was supported by DARPA MTO under the EPHI contract and the FP7-ERC-InSpectra project. Andreas De Groote thanks the research foundation Flanders (FWO) for a research grant and also the Belgian American Educational Foundation (BAEF) for the support.

\section{REFERENCES}

[1] S. Keyvaninia et. al., Opt. Lett. 38, 5434-5437 (2013).

[2] M. Heck et. al., IEEE J. Sel. Top. Quantum Electron. 19, (2013).

[3] G. Roelkens, Laser Photon. Rev 4, 751-779 (2010).

[4] B.-R. Wu, Electron. Lett. 36, 2093-2095 (2000).

[5] JH. Song, IEEE Photon. Technol. Lett 12, 783-785 (2000).

[6] A. Kovsh, Photonics Europe, 69960V-69960V (2008).

[7] S. Uvin, IEEE Photonics Conference, (2013).

[8] SR. Jain, j. Lightw. Technol. 30, 671-678 (2012).

[9] F. Riesz, Mater. Sci. Eng. B 80, 54-59 (2001).

[10] AW. Fang, Mater. Today 10, 28-35 (2007).

[11] C. Zhang, Opt. Express 22, 10202-10209 (2014). 